\title{
Grau de dependência à nicotina de pacientes atendidos para tratamento do tabagismo em universidade pública
}

Rafael da Rosa Maciel ${ }^{1}$

(D) https://orcid.org/0000-0003-3146-9103

Lidia Dalgallo ${ }^{1}$

(D) https://orcid.org/0000-0003-1328-9483

Erildo Vicente Müller ${ }^{1}$

(1D) https://orcid.org/0000-0003-4643-056X

Elaine Cristina Antunes Rinaldi

(D) https://orcid.org/0000-0002-2937-7405

Universidade Estadual de Ponta Grossa, Campus Uvaranas, Ponta Grossa, PR, Brasil.

\begin{abstract}
Objetivo: identificar o grau de dependência à nicotina e dados sociodemográficos segundo o gênero dos pacientes atendidos pelo projeto de extensão Educando e Tratando o Tabagismo promovido por uma universidade pública. Método: estudo transversal, com dados provenientes de 361 prontuários de pacientes que participantes do projeto no período de 2014 a 2018. Realizou-se o teste de Fagerström, por meio do qual classificou-se os pacientes com grau de dependência: muito baixo, baixo, médio, elevado e muito elevado de acordo com a soma dos pontos obtidos nas questões aplicadas. Resultados: a predominância no grau de dependência a nicotina foi observada no sexo feminino com maiores percentuais de classificação para dependência elevada e muito elevada, $33,5 \%$ e $25,3 \%$ respectivamente. Entre os homens verificouse grau de dependência elevado $28,1 \%$ e baixo $21,9 \%$. Contatou-se que o grau de dependência química a nicotina esteve associada a dependência física (valor- $p=0,002$ ) e psicológica (valor-p=0,003). Conclusão: a evidência de maior frequência de indivíduos com alto grau de dependência química à nicotina demonstra a importância da associação de terapias medicamentosas com abordagens cognitivas comportamentais por meio de intervenções de enfermagem, relacionadas ao controle e cessação do tabagismo por meio da educação em saúde.
\end{abstract}

Descritores: Dependência Química; Fumar; Nicotina; Tabagismo.

\section{Como citar este artigo}

Maciel RR, Dalgallo L, Müller EV, Rinaldi ECA. Nicotine dependence degree of patients treated for smoking addiction at a public university. SMAD, Rev Eletrônica Saúde Mental Álcool Drog. 2021 jan.- mar.;17(1):48-57. doi: https://dx.doi.org/10.11606/issn.1806-6976.smad.2021.163327 


\section{Nicotine dependence degree of patients treated for smoking addiction at a public university}

Objective: to identify the nicotine dependence degree and sociodemographic data according to the gender of the patients assisted by the Educating and Treating Smoking extension project promoted by a public university. Method: a cross-sectional study, with data from 361 medical records of patients participating in the project from 2014 to 2018 . The Fagerström test was performed, through which patients with a degree of dependence were classified as follows: very low, low, medium, high, and very high, according to the sum of the points obtained in the applied questions. Results: predominance in the nicotine dependence degree was observed in women with higher percentages of classification for high and very high dependence, $33.5 \%$ and $25.3 \%$ respectively. Among men, a high degree of dependence was found to be $28.1 \%$ and low, $21.9 \%$. It was found that the degree of chemical dependence on nicotine was associated with physical $(p$-value $=0.002)$ and psychological ( $p$-value $=0.003)$ dependence. Conclusion: the evidence for a higher frequency of individuals with a high chemical dependence degree for nicotine demonstrates the importance of combining drug therapies with cognitive behavioral approaches through Nursing interventions, related to controlling and quitting the smoking addiction through health education.

Descriptors: Chemical Dependency; Smoking; Nicotine; Tobacco use Disorder.

\section{Grado de dependencia a la nicotina de pacientes tratados por fumar en una universidad pública}

Objetivo: identificar el grado de dependencia a la nicotina y los datos sociodemográficos según el género de los pacientes atendidos por el proyecto de extensión Educar y tratar el tabaquismo promovido por una universidad pública. Método: estudio transversal, con datos de 361 registros médicos de pacientes que participaron en el proyecto de 2014 a 2018. Se realizó la prueba de Fagerström, a través de la cual se clasificaron los pacientes con un grado de dependencia: muy bajo, bajo, medio, alto y muy alto de acuerdo con la suma de los puntos obtenidos en las preguntas aplicadas. Resultados: el predominio en el grado de dependencia a la nicotina se observó en mujeres con porcentajes más altos de clasificación de dependencia alta y muy alta, 33.5\% y $25.3 \%$ respectivamente. Entre los hombres, se encontró un alto grado de dependencia del 28,1\% y bajo del 21,9\%. Se encontró que el grado de dependencia química de la nicotina se asoció con el físico (valor $p=0.002$ ) y psicológico (valor $p=0.003$ ). Conclusión: la evidencia de una mayor frecuencia de individuos con un alto grado de dependencia química de la nicotina demuestra la importancia de combinar las terapias farmacológicas con enfoques cognitivos conductuales a través de intervenciones de enfermería, relacionadas con el control del tabaquismo y la cesación a través de la educación sanitaria.

Descriptores: Dependencia Química; Fumar; Nicotina; Tabaquismo. 


\section{Introdução}

O tabagismo é considerado uma das principais causas de mortes evitáveis no mundo e colabora negativamente para o crescente problema na saúde pública, pois apresenta um acentuado risco de desenvolvimento de câncer, problemas respiratórios, cardiovasculares, dentre outros(1).

A ação do tabaco provoca milhões de mortes e, caso não sejam tomadas devidas providências, esse quantitativo tende a crescer visto que o número de fumantes tem aumentado cada vez mais, principalmente em países em desenvolvimento(2).

A principal substância responsável pela dependência do tabagismo é a nicotina. Conforme estudos, aproximadamente $90 \%$ dos fumantes diários são dependentes dela, uma vez que cerca de $25 \%$ da nicotina inalada atravessa os alvéolos pulmonares e chega ao encéfalo via sanguínea em 15 segundos, fato este que corrobora para o alto índice de dependência ser causada rapidamente ${ }^{(3-4)}$.

Há a ocorrência de dois tipos de dependência química, a física, onde acontecem os sinais de abstinência, como por exemplo sudorese e tremores e a dependência psicológica, onde os indivíduos tabagistas buscam no fumo como uma válvula de escape para os problemas do dia a dia, utilizando o cigarro como uma forma de acalmar-se e aliviar o estresse(5).

O teste de Fagerström, reconhecido mundialmente, é utilizado para avaliar a dependência à nicotina. É por meio deste que se estabelece o grau de dependência relacionada à nicotina, sendo classificada de acordo com o resultado obtido em: muito baixo, baixo, médio, elevado e muito elevado(4).

Estudos retratam que o grau de dependência à nicotina influenciará diretamente na maior ou menor dificuldade de o indivíduo abandonar o vício, o que pode comprometer significativamente a saúde ${ }^{(6)}$.

Deste modo, avaliar o grau de dependência relacionado à nicotina é de suma importância, pois é a partir desta substância que os indivíduos se tornam dependentes do tabaco, o que os leva a ter problemas sérios tanto relacionado à saúde, quanto nos aspectos biopsicossociais, colaborando para um déficit da qualidade de vida.

Diante deste contexto, tem-se a problemática: de que forma o grau de dependência à nicotina afeta na cessação do tabaco nos pacientes que fazem tratamento?

Neste estudo, objetiva-se identificar o grau de dependência à nicotina e os dados sociodemográficos segundo o gênero dos pacientes atendidos pelo projeto de extensão Educando e Tratando o Tabagismo da Universidade Estadual de Ponta Grossa (UEPG) no Paraná (PR).

\section{Método}

Trata-se de um estudo epidemiológico transversal, com dados obtidos a partir das fichas dos pacientes que participaram no período de 2014 a 2018 no Projeto de Extensão intitulado Educando e Tratando o Tabagismo, localizado na Universidade Estadual de Ponta Grossa, PR. $O$ projeto tem por finalidade tratar o tabagismo e segue as normas estabelecidas pelo Instituto Nacional de Câncer (INCA) e o Ministério da Saúde.

O desenvolvimento do projeto na Universidade, ocorre por meio de quatro encontros semanais e três encontros quinzenais de manutenção, num primeiro momento é realizada a abordagem cognitivo comportamental, em grupo. Esta assistência é realizada pelos graduandos de enfermagem, educação física, farmácia, medicina e professores. Em um segundo momento os pacientes são atendidos individualmente por consulta de enfermagem, educação física e médica, a qual estabelece o tratamento medicamentoso, quando necessário, com o objetivo de auxiliar no tratamento do tabagismo. Medicamentos estes que são distribuídos gratuitamente, pois fazem parte do programa ministerial, disponibilizados na farmácia escola da Universidade Estadual de Ponta Grossa.

As variáveis de interesse estão representadas pelo grau de d ependência à nicotina, sendo obtida por meio do teste de Fagerström, que classifica a dependência dos indivíduos em muito baixo ( 0 a 2), baixo (3 a 4), médio (5), elevado ( 6 a 7) e muito elevado ( 8 a 10), de acordo com a pontuação obtida no final do teste. Para as variáveis sociodemográficas, foram elencadas idade, sexo, escolaridade, estado civil, tipo de dependência, grau de motivação e método escolhido para cessação do fumo, tipo de medicamentos utilizados, se anteriormente fez tratamento ou parte de algum grupo de tratamento do tabagismo, o número de cigarros consumidos por dia e também o tempo de uso do fumo. E por fim, a porcentagem da cessação do tabaco, que é obtida no último encontro após avaliação médica.

A coleta de dados foi realizada na Universidade, junto aos prontuários dos pacientes, no período de novembro e dezembro de 2018. Inicialmente, ocorreu a digitação dos dados em planilha do software Excel, logo após procedeu-se as análises descritivas com estimativas de frequências simples e relativas das variáveis de interesse de acordo com a classificação em nível de dependência baixo/muito baixo, moderado e elevado/muito elevado. Para verificar as diferenças entre as proporções das variáveis qualitativas de acordo com estas classificações, foi aplicado o teste de qui-quadrado. Para melhor visualização das análises qualitativas, foram produzidos gráficos de barras com as frequências relativas.

Para as variáveis quantitativas idade, número de cigarros/dia e anos de fumo, foi realizado o teste 
Shapiro-Wilk para aderência à distribuição normal e, dado que não houve simetria na distribuição das mesmas (valor-p > 0,05), optou-se pela abordagem não paramétrica dos dados. Então, utilizou-se a prova de Kruskall-Wallys, seguido de Dunn como post-hoc para verificar as diferenças nos níveis de dependência. Para melhor visualização das análises quantitativas, foram produzidos gráficos do tipo boxplot. Todos os testes foram considerados significativos quando valor-p $<0,05$ e as análises foram realizadas na plataforma de software IBM SPSS Statistics 20.0.

A pesquisa obteve aprovação do comitê de ética em pesquisa da Universidade Estadual de Ponta Grossa no ano de 2018 sob o número 2.991.057.

\section{Resultados}

Entre os anos de 2014 a 2018, participaram do projeto de extensão Educando e Tratando o Tabagismo da Universidade Estadual de Ponta Grossa um total de 361 indivíduos, observa-se que a procura pelo projeto foi predominantemente por mulheres, correspondendo a $64,5 \%$ dos participantes.

Na Tabela 1 descreve-se a escolaridade e idade média dos pacientes. Predominou-se entre os homens o ensino médio completo e superior completo ou incompleto, com $35,2 \%$ e $27,3 \%$ respectivamente. Para as mulheres observa-se maior frequência de ensino fundamental incompleto $30,5 \%$ e médio completo $37,8 \%$. Os homens tiveram a média de idade de 45,8 anos (Desvio Padrão $=14,1$ ) e entre as mulheres a média foi de 49,5 anos (Desvio Padrão $=11,8$ ).

Tabela 1 - Escolaridade e idade média dos participantes do Projeto de Extensão Educando e Tratando o Tabagismo promovido no período de 2014 a 2018 pela Universidade Estadual de Ponta Grossa. Ponta Grossa, PR, Brasil, 2018

\begin{tabular}{|c|c|c|c|c|}
\hline \multirow{2}{*}{ Variáveis } & \multicolumn{2}{|c|}{ Homens } & \multicolumn{2}{|c|}{ Mulheres } \\
\hline & Total & $\%$ & Total & $\%$ \\
\hline \multicolumn{5}{|l|}{1 - Nível de escolaridade } \\
\hline Ensino fundamental incompleto & 31 & $24,2 \%$ & 71 & $30,5 \%$ \\
\hline Ensino fundamental completo & 11 & $8,6 \%$ & 27 & $11,6 \%$ \\
\hline Ensino médio incompleto & 06 & $4,7 \%$ & 11 & $4,7 \%$ \\
\hline Ensino médio completo & 45 & $35,2 \%$ & 88 & $37,8 \%$ \\
\hline Ensino superior incompleto/completo & 35 & $27,3 \%$ & 36 & $15,4 \%$ \\
\hline \multirow[t]{2}{*}{ TOTAL } & 128 & $100 \%$ & 233 & $100 \%$ \\
\hline & Média & Desvio padrão & Média & $\begin{array}{l}\text { Desvio } \\
\text { padrão }\end{array}$ \\
\hline 2 - Idade & 45,8 & 14,1 & 45,9 & 11,8 \\
\hline
\end{tabular}

A Tabela 2 refere-se as características dos tabagistas que participaram do Projeto de Extensão Educando e Tratando o Tabagismo. No que diz respeito ao tipo de dependência à nicotina, foi mais frequente a física tanto para homens, quanto para mulheres com $49,2 \%$ e $55,4 \%$ respectivamente.

Tabela 2 - Características dos tabagistas participantes do Projeto de Extensão Educando e Tratando o Tabagismo promovido no período de 2014 a 2018 pela Universidade Estadual de Ponta Grossa. Ponta Grossa, PR, Brasil, 2018

\begin{tabular}{|c|c|c|c|c|}
\hline \multirow{2}{*}{ Variáveis } & \multicolumn{2}{|c|}{ Homens } & \multicolumn{2}{|c|}{ Mulheres } \\
\hline & Total & $\%$ & Total & $\%$ \\
\hline \multicolumn{5}{|l|}{1 - Tipo de dependência } \\
\hline Não informado & 54 & $42,2 \%$ & 106 & $45,5 \%$ \\
\hline Fisicamente dependente & 63 & $49,2 \%$ & 129 & $55,4 \%$ \\
\hline Associação de comportamento & 59 & $46,1 \%$ & 86 & $36,9 \%$ \\
\hline Psicologicamente dependente & 39 & $30,5 \%$ & 74 & $31,8 \%$ \\
\hline \multicolumn{5}{|l|}{2 - Grau de motivação para a cessação do fumo } \\
\hline Não informado & 63 & $49,2 \%$ & 121 & $51,9 \%$ \\
\hline Pré contemplação: não pensa em parar de fumar & 0 & $0 \%$ & 02 & $0,9 \%$ \\
\hline Contemplação: pensa em parar de fumar algum dia & 09 & $7,0 \%$ & 17 & $7,2 \%$ \\
\hline Preparação: pensa em marcar data para cessar o fumo & 12 & $9,4 \%$ & 21 & $9,0 \%$ \\
\hline Pronto para agir: quer cessar o fumo no primeiro mês & 35 & $27,4 \%$ & 58 & $24,9 \%$ \\
\hline Manutenção: parou de fumar & 0 & $0 \%$ & 02 & $0,9 \%$ \\
\hline Recaída: parou de fumar, mas recaiu & 09 & $7,0 \%$ & 12 & $5,2 \%$ \\
\hline \multicolumn{5}{|l|}{3 - Método escolhido para cessação do tabagismo } \\
\hline Não informado & 33 & $25,8 \%$ & 61 & $26,2 \%$ \\
\hline Parada abrupta & 39 & $30,5 \%$ & 60 & $25,8 \%$ \\
\hline Parada gradual & 49 & $38,3 \%$ & 97 & $41,6 \%$ \\
\hline
\end{tabular}


(Tabela 2 - continuação)

\begin{tabular}{|c|c|c|c|c|}
\hline \multirow{2}{*}{ Variáveis } & \multicolumn{2}{|c|}{ Homens } & \multicolumn{2}{|c|}{ Mulheres } \\
\hline & Total & $\%$ & Total & $\%$ \\
\hline Apenas redução & 04 & $3,1 \%$ & 13 & $5,6 \%$ \\
\hline Adiamento & 03 & $2,3 \%$ & 02 & $0,8 \%$ \\
\hline \multicolumn{5}{|c|}{4 - Medicamentos utilizados para cessação do tabagismo } \\
\hline Não utilizou medicamento & 21 & $16,4 \%$ & 20 & $8,6 \%$ \\
\hline Adesivo de nicotina & 104 & $81,3 \%$ & 210 & $90,1 \%$ \\
\hline Bupropiona & 25 & $19,5 \%$ & 41 & $17,6 \%$ \\
\hline Terapia combinada & 21 & $16,4 \%$ & 38 & $16,3 \%$ \\
\hline \multicolumn{5}{|c|}{5 - Desistência do projeto, redução e cessação do uso do cigarro } \\
\hline Desistência & 38 & $29,7 \%$ & 66 & $28,3 \%$ \\
\hline Redução & 44 & $34,4 \%$ & 86 & $36,9 \%$ \\
\hline Cessação & 46 & $35,9 \%$ & 81 & $34,8 \%$ \\
\hline \multicolumn{5}{|c|}{6 - Já fez tratamento para cessação do fumo } \\
\hline Sim & 31 & $24,2 \%$ & 76 & $32,6 \%$ \\
\hline Não & 97 & $75,8 \%$ & 156 & $67,4 \%$ \\
\hline \multicolumn{5}{|c|}{7 - Já fez parte de algum grupo de apoio que tratasse o tabagismo } \\
\hline $\operatorname{Sim}$ & 11 & $8,6 \%$ & 40 & $17,2 \%$ \\
\hline Não & 117 & $91,4 \%$ & 193 & $82,8 \%$ \\
\hline \multirow[t]{2}{*}{ TOTAL } & 128 & - & 233 & - \\
\hline & Média & $\begin{array}{l}\text { Desvio } \\
\text { padrão }\end{array}$ & Média & $\begin{array}{l}\text { Desvio } \\
\text { padrão }\end{array}$ \\
\hline 8 - Cigarros consumidos por dia & 24,2 & 9,2 & 23,0 & 9,0 \\
\hline 9 - Tempo de fumo em anos & 27,7 & 14,5 & 31,4 & 13,1 \\
\hline
\end{tabular}

Observou-se entre ambos os sexos maior prevalência de estar pronto para parar de fumar nas primeiras quatro semanas com $27,4 \%$ para os homens e $24,9 \%$ para as mulheres. Percebe-se que o método escolhido para cessação do tabaco que se destacou foi a parada gradual com $38,3 \%$ para homens e $41,6 \%$ para as mulheres. Verificou-se que tanto homens, quanto mulheres utilizaram para terapia medicamentosa o adesivo de nicotina para a cessação do fumo, com $81,3 \%$ e $90,1 \%$ respectivamente.

Constatou-se que $35,9 \%$ dos homens e $32,8 \%$ das mulheres cessaram o tabaco. Com relação a redução do consumo de cigarros por dia, cerca de $24,4 \%$ dos homens conseguiram reduzir. Entre as mulheres ocorreu um maior índice de redução, em torno de 36,9\%. Quanto a taxa de abandono do tratamento, pelos homens foi de $29,7 \%$ e mulheres $28,3 \%$.

Aproximadamente $75,8 \%$ dos homens e $67,4 \%$ das mulheres afirmaram nunca ter realizado algum tipo de tratamento para o tabagismo. E também entre os homens e mulheres, a grande maioria, $91,4 \%$ e $82,8 \%$ respectivamente, afirmaram nunca ter participado de um grupo de apoio para o tratamento do tabagismo.

Destaca-se ainda que a média de consumo de cigarros pelos homens foi em média cerca de 24,2 cigarros por dia, (Desvio Padrão $=9,2$ ). Entre as mulheres, observou-se a média de consumo de 23,0 cigarros por dia (Desvio Padrão $=9,0$ ). Quanto ao tempo de fumo dos pacientes, os homens tiveram em média 27,7 anos (Desvio Padrão $=14,5$ ) e as mulheres média de 31,4 anos (Desvio Padrão $=13,1$ ).

O resultado do teste de Fagerström, está exposto na Tabela 3. Observou- se maior prevalência entre as mulheres do grau elevado $33,5 \%$ e muito elevado $25,3 \%$. Entre os homens, verificou-se maior predomínio dos graus elevado e baixo, com $28,1 \%$ e $21,9 \%$ respectivamente.

Tabela 3 - Teste de Fagerström aplicado nos participantes do Projeto de Extensão Educando e Tratando do Tabagismo promovido no período de 2014 a 2018 pela Universidade Estadual de Ponta Grossa. Ponta Grossa, PR, Brasil, 2018

\begin{tabular}{lcccc}
\hline \multirow{2}{*}{ Grau de dependência } & \multicolumn{2}{c}{ Mulheres } & \multicolumn{2}{c}{ Homens } \\
\cline { 2 - 5 } & Total & $\%$ & Total & $\%$ \\
\hline Muito baixo & 18 & $7,7 \%$ & 12 & $9,4 \%$ \\
Baixo & 43 & $18,5 \%$ & 25 & $21,9 \%$ \\
Médio & 35 & $15,0 \%$ & 26 & $20,3 \%$ \\
Elevado & 78 & $33,5 \%$ & 36 & $28,1 \%$ \\
Muito Elevado & 59 & $25,3 \%$ & 26 & $20,3 \%$ \\
\hline TOTAL & 233 & $100 \%$ & 128 & $100 \%$ \\
\hline
\end{tabular}

A associação entre grau de dependência à nicotina, variáveis sociodemográficas e características dos tabagistas estão expostas na Tabela 4. Verifica-se que alto grau de dependência a nicotina esteve associado a dependência física (valor-p =0,002) e psicológica (valor-p $=0,003)$. 
Tabela 4 - Associação entre variáveis sociodemográficas, as características dos tabagistas e o grau de dependência à nicotina. Ponta Grossa, PR, Brasil, 2018

\begin{tabular}{|c|c|c|c|c|c|c|c|c|c|}
\hline \multirow{3}{*}{ Variáveis } & \multicolumn{6}{|c|}{ Grau de dependência química } & & & \multirow{3}{*}{ P-valor } \\
\hline & \multicolumn{2}{|c|}{ Baixo e muito baixo } & \multicolumn{2}{|c|}{$\begin{array}{c}\text { Elevado e muito } \\
\text { elevado }\end{array}$} & \multicolumn{2}{|c|}{ Médio } & \multicolumn{2}{|c|}{ Total } & \\
\hline & $\mathbf{n}$ & $\%$ & n & $\%$ & $\mathbf{n}$ & $\%$ & n & $\%$ & \\
\hline \multicolumn{10}{|l|}{ Sexo } \\
\hline Masculino & 31 & $29,5 \%$ & 50 & $47,6 \%$ & 24 & $22,9 \%$ & 105 & $100 \%$ & \multirow{2}{*}{0,998} \\
\hline Feminino & 46 & $23,8 \%$ & 19 & $61,7 \%$ & 28 & $14,5 \%$ & 90 & $100 \%$ & \\
\hline \multicolumn{10}{|l|}{ Estado civil } \\
\hline Viúvo & 6 & $23,1 \%$ & 18 & $69,2 \%$ & 2 & $7,7 \%$ & 26 & $100 \%$ & \multirow{5}{*}{0,233} \\
\hline Solteiro & 23 & $32,9 \%$ & 35 & $50,0 \%$ & 12 & $17,1 \%$ & 70 & $100 \%$ & \\
\hline Divorciado & 5 & $17,2 \%$ & 21 & $72,4 \%$ & 3 & $10,3 \%$ & 29 & $100 \%$ & \\
\hline Casado/união estável & 36 & $24,0 \%$ & 81 & $54,0 \%$ & 33 & $22,0 \%$ & 150 & $100 \%$ & \\
\hline Não informado & 7 & $30,4 \%$ & 14 & $60,9 \%$ & 2 & $8,7 \%$ & 23 & $100 \%$ & \\
\hline \multicolumn{10}{|l|}{ Escolaridade } \\
\hline Analfabeto & 0 & $0,0 \%$ & 1 & $100 \%$ & 0 & $0,0 \%$ & 1 & $100 \%$ & \multirow{5}{*}{0,728} \\
\hline Ensino fundamental & 29 & $27,4 \%$ & 58 & $54,7 \%$ & 19 & $17,9 \%$ & 106 & $100 \%$ & \\
\hline Ensino médio & 28 & $26,7 \%$ & 60 & $57,1 \%$ & 17 & $16,2 \%$ & 105 & $100 \%$ & \\
\hline Ensino superior & 14 & $23,7 \%$ & 31 & $52,5 \%$ & 14 & $23,7 \%$ & 59 & $100 \%$ & \\
\hline Não informado & 6 & $22,2 \%$ & 19 & $70,4 \%$ & 2 & $7,4 \%$ & 27 & $100 \%$ & \\
\hline \multicolumn{10}{|l|}{ Fisicamente dependente } \\
\hline Sim & 24 & $17,8 \%$ & 91 & $67,4 \%$ & 20 & $14,8 \%$ & 135 & $100 \%$ & \multirow{2}{*}{0,002} \\
\hline Não & 53 & $32,5 \%$ & 78 & $47,9 \%$ & 32 & $19,6 \%$ & 163 & $100 \%$ & \\
\hline \multicolumn{10}{|c|}{ Associações de comportamento } \\
\hline Sim & 29 & $24,4 \%$ & 70 & $58,8 \%$ & 20 & $16,8 \%$ & 119 & $100 \%$ & \multirow{2}{*}{0,832} \\
\hline Não & 48 & $26,8 \%$ & 99 & $55,3 \%$ & 32 & $17,9 \%$ & 179 & $100 \%$ & \\
\hline \multicolumn{10}{|c|}{ Psicologicamente dependente } \\
\hline Sim & 15 & $16,3 \%$ & 68 & $73,9 \%$ & 9 & $9,8 \%$ & 92 & $100 \%$ & \multirow{2}{*}{0,003} \\
\hline Não & 62 & $30,1 \%$ & 101 & $49,0 \%$ & 43 & $20,9 \%$ & 206 & $100 \%$ & \\
\hline \multicolumn{10}{|l|}{ Parada abrupta } \\
\hline Sim & 20 & $27,0 \%$ & 42 & $56,8 \%$ & 12 & $16,2 \%$ & 74 & $100 \%$ & \multirow{2}{*}{0,933} \\
\hline Não & 57 & $25,4 \%$ & 127 & $56,7 \%$ & 40 & $17,9 \%$ & 224 & $100 \%$ & \\
\hline \multicolumn{10}{|l|}{ Parada gradual } \\
\hline Sim & 23 & $20,4 \%$ & 67 & $59,3 \%$ & 23 & $20,4 \%$ & 113 & $100 \%$ & 0.201 \\
\hline Não & 54 & $29,2 \%$ & 102 & $55,1 \%$ & 29 & $15,7 \%$ & 185 & $100 \%$ & ו ו \\
\hline Apenas redução & & & & & & & & & \\
\hline Sim & 4 & $16,7 \%$ & 15 & $62,5 \%$ & 5 & $20,8 \%$ & 24 & $100 \%$ & 0556 \\
\hline Não & 73 & $26,6 \%$ & 154 & $56,2 \%$ & 47 & $17,2 \%$ & 274 & $100 \%$ & 0,000 \\
\hline Adiamento & & & & & & & & & \\
\hline Sim & 1 & $14,3 \%$ & 6 & $85,7 \%$ & 0 & $0,0 \%$ & 7 & $100 \%$ & 0261 \\
\hline Não & 76 & $26,1 \%$ & 163 & $56,0 \%$ & 52 & $17,9 \%$ & 291 & $100 \%$ & $0, \angle 01$ \\
\hline Medicação: adesivo & & & & & & & & & \\
\hline Não & 12 & $30,0 \%$ & 21 & $52,5 \%$ & 7 & $17,5 \%$ & 40 & $100 \%$ & 0806 \\
\hline Sim & 65 & $25,4 \%$ & 147 & $57,4 \%$ & 44 & $17,2 \%$ & 256 & $100 \%$ & U, ర00 \\
\hline Medicação: bupropiona & & & & & & & & & \\
\hline Não & 70 & $27,2 \%$ & 143 & $55,6 \%$ & 44 & $17,1 \%$ & 257 & $100 \%$ & \\
\hline Sim & 7 & $17,9 \%$ & 25 & $64,1 \%$ & 7 & $17,9 \%$ & 39 & $100 \%$ & 0,400 \\
\hline
\end{tabular}

A associação entre o grau de dependência à nicotina, variáveis sociodemográficas e características do tabagismo está descrito na Tabela 5 . Verifica-se que quanto maior a mediana de cigarros consumidos por dia, maior é o grau de dependência a nicotina (valor-p $=0,003$ ). 
Tabela 5 - Associação entre o grau de dependência à nicotina, idade, cigarros consumidos por dia e anos de fumo dos participantes do Projeto de Extensão Educando e Tratando o Tabagismo promovido no período de 2014 a 2018 pela Universidade Estadual de Ponta Grossa. Ponta Grossa, PR, Brasil, 2018

\begin{tabular}{lcccc}
\hline $\begin{array}{l}\text { Grau de } \\
\text { dependência }\end{array}$ & Estatística & Idade & $\begin{array}{c}\text { Cigarros/ } \\
\text { dia }\end{array}$ & $\begin{array}{c}\text { Anos de } \\
\text { fumo }\end{array}$ \\
\hline & Média & 47 & 17 & 29 \\
Baixo e muito & Desvio padrão & 14 & 5 & 15 \\
baixo & Mediana & 49 & 20 & 30 \\
& $\begin{array}{c}\text { Intervalo } \\
\text { interquartil }\end{array}$ & $8-56$ & $10-20$ & $20-40$ \\
& Média & 47 & 28 & 30 \\
Elevado e muito & $\begin{array}{c}\text { Desvio padrão } \\
\text { elevado }\end{array}$ & 12 & 9 & 13 \\
& Mediana & 49 & 30 & 30 \\
& $\begin{array}{c}\text { Intervalo } \\
\text { interquartil }\end{array}$ & $37-57$ & $20-40$ & $20-40$ \\
& Média & 50 & 21 & 30 \\
& Desvio padrão & 13 & 7 & 14 \\
\hline Médio & Mediana & 52 & 20 & 21 \\
& $\begin{array}{c}\text { Intervalo } \\
\text { interquartil }\end{array}$ & $44-27$ & $20-20$ & $21-20$ \\
\hline
\end{tabular}

\section{Discussão}

Os homens são os maiores consumidores de tabaco ao redor do mundo(7). No entanto, na presente pesquisa ocorreu predomínio de mulheres tabagistas a procura de tratamento, porém, deve-se ressaltar que historicamente homens procuram por tratamento ou apoio somente quando os sintomas de enfermidades estão mais evidentes, fato esse que pode ter contribuído para a baixa demanda do público masculino para cessar o uso do tabaco(8). $^{(8)}$.

Contrastando com estudos semelhantes, apresentaram-se principalmente tabagistas com baixa escolaridade, prevalecendo o ensino fundamental e médio incompleto(9-11). Este é um dos fatores que podem influenciar no sucesso ou não da cessação do tabaco, uma vez que indivíduos com menos anos de estudo apresentam maior dificuldade para cessar o vício devido ao baixo grau de instrução por eles adquiridos no decorrer dos

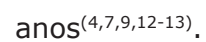

No que se refere a idade média dos tabagistas, o resultado foi equivalente a estudos anteriores, que relatam ter cerca de 45 anos tanto para homens, quanto para mulheres ${ }^{(11-12,14)}$. Há o apontamento em pesquisas que a idade de iniciação do tabagismo está cada vez mais precoce, em torno de 15 anos de idade aproximadamente ${ }^{(10,15)}$. Sabe-se que a idade precoce influencia no tempo médio de fumo dos tabagistas que, de acordo com estudos está em torno de 30 anos, o que expressa um reflexo desta pesquisa e também apresenta maiores riscos pois, as chances de desenvolver doenças associadas ao tabaco aumentam significativamente ${ }^{(16-17)}$.
Contudo, uma pesquisa recente vem apontando a diminuição da prevalência do tabagismo entre os jovens brasileiros, fato este que é extremamente importante para a melhor qualidade de vida dos indivíduos ${ }^{(18)}$.

Há um déficit de estudos na área relacionada ao tipo de dependência, seja ela física ou psicológica. Os dependentes fisicamente à nicotina despontaram com maior frequência neste estudo, o que pode ser considerado um sinal de alerta, pois as chances dos sinais de abstinência se desenvolver é maior devido ao alto grau de dependência relacionada à nicotina. Enquanto que os tabagistas considerados psicologicamente dependentes à nicotina buscam o fumo como uma válvula de escape para o estresse e problemas do dia a dia isso pode-se tornar rotineiro de modo que não percebam que estão se tornando cada vez mais dependentes(5).

Do mesmo modo que evidenciado por um estudo análogo, predominou com maior frequência a fase motivacional pronto para a ação e isto demonstra a vontade de querer cessar o tabaco nas primeiras quatro semanas de início do tratamento, o que é muito significativo para conseguir o objetivo principal que é eliminar o vício à nicotina, por outro lado o tipo de acolhimento dos profissionais de saúde para tal fato é muito importante, pois quanto mais demonstrar apoio, maior será a motivação para não abandonar o tratamento(19).

De maneira pariforme a outros estudos, o modo abrupto, que é aquele em que o tabagista para repentinamente de fumar, e a parada gradual, na qual o fumante vai gradativamente diminuindo os números de cigarros/dia, são geralmente os mais escolhidos para cessação do fumo(20-21).

A famacoterapia é utilizada para o controle da abstinência relacionada a nicotina, sendo majoritariamente utilizados o adesivo de nicotina e a bupropiona(22). A literatura nacional tem mostrado quem entre tais medicações, o adesivo de nicotina é em larga escala o mais empregado para o tratamento do tabagismo(23).

Devido a muitas vezes o grau de dependência química à nicotina estar muito alta, ocorrem desistências do tratamento do tabagismo e, para aqueles indivíduos que não conseguiram cessar o fumo na primeira vez, já é relevante a participação e o breve conhecimento sobre o tratamento, evidenciando que os profissionais de saúde têm um papel importantíssimo em engajar e apoiar cada vez mais as pessoas que queiram cessar o fumo ${ }^{(15)}$.

Várias conjecturas apontam que os tabagistas, principalmente àqueles com alto grau de dependência à nicotina jamais fizeram tratamento para parar de fumar pois, devido ao vício ser grande, a vontade de tentar cessar o fumo é quase que inexistente o que pode comprometer o sucesso no tratamento se não for abordado de forma adequada(24-25). 
A carga tabagística é algo que está estritamente ligado a elevada dependência à nicotina e também aos aspectos econômicos, uma vez que o alto custo para adquirir cigarros diariamente poderia ser revertido para suprir outras necessidades essenciais ${ }^{(26)}$. Neste estudo, a média de cigarros consumidos por dia foi entre 22 e 24, fato este que corrobora com pesquisas nacionais que apontam os mesmos resultados no decorrer das análises estatísticas ${ }^{(26-28)}$.

Há na literatura incontáveis pesquisas acerca da grande parcela de tabagistas que são considerados segundo o teste de Fagerström com a dependência à nicotina elevada e muito elevada, necessitando além de um alto grau de motivação, uma abordagem cognitiva comportamental e também medicamentosa para a cessação do tabagismo(6,14,16,27,29).

O presente estudo permitiu o conhecimento do perfil sociodemográfico e de dependência dos indivíduos atendidos pelo programa Educando e Tratando o Tabagismo, e pode subsidiar ações para melhorar a abordagem e intervenções futuras que impactem diretamente na cessação do tabagismo.

As limitações encontradas se referiram ao não preenchimento de algumas informações, apontando assim a necessidade de calibração da equipe executora do projeto.

Outro fator a ser mencionado é que o número de tabagistas que cessaram definitivamente o tabaco pode ser maior que o apresentado pois, podem ter conseguido cessar após o término do tratamento no Projeto, porém essa informação não foi coletada.

\section{Conclusão}

O estudo possibilitou identificar o grau de dependência à nicotina e os dados sociodemográficos segundo o gênero dos pacientes atendidos pelo projeto de extensão Educando e Tratando o Tabagismo da Universidade Estadual de Ponta Grossa-PR.

Grande parte dos indivíduos tabagistas caracteriza-se pela alta taxa de cigarros consumidos por dia em conjunção com a dependência tanto física, quanto psicológica, fato este, que associado muitas vezes a baixa escolaridade, contribui negativamente para que o grau de dependência relacionada à nicotina seja extremamente alto.

Independente do gênero, houve maior frequência de alto grau de dependência à nicotina nos pacientes demonstrando a importância da associação de terapias medicamentosas com abordagens cognitivas comportamentais por meio de intervenções de enfermagem, relacionadas ao controle e cessação do tabagismo por meio da educação em saúde.

\section{Referências}

1. Lima MBP, Ramos D, Freire APCF, Uzeloto JS, Silva BLM, Ramos EMC. Quality of life of smokers and its correlation with smoke load. Fisioter Pesqui. 2017; 24(3):273-9. doi: https://doi.org/10.1590/1809-2950/16711324032017 2. Costa SCR, Feitosa SDM, Neves VC, Costa MFS, Sá LLF, Veloso RP. Revisão bibliográfica: políticas públicas do tabagismo no Brasil. J Ciênc Saúde. 2018;1(2):97-104. doi: https://doi. org/10.26694/2595-0290.20181297-1047113

3. Pupulim AF, Sarris AB, Fernandes LGR, Nakamura MC, Camargo TV, Paula JB. Mecanismos de dependência química no tabagismo: revisão da literatura. Rev Med UFPR. 2015;2(2):74-8. doi: http://dx.doi.org/10.5380/ rmu.v2i2.42122.g25672

4. Weber CF, Hatschbach P, Pithan SA, Dullius AIS. Measure nicotine dependence by the fagerström test for nicotine dependence. Rev Gaúch Odontol. 2017;65(3):208-15. doi: https://doi. org/10.1590/1981-863720170002000043223

5. Fernandes LS, Nitsche MJT, Godoy I. Association between burnout syndrome, harmful use of alcohol and smoking in nursing in the ICU of a university hospital. Ciênc Saúde Coletiva. 2018;23(1):203-14. doi: https:// doi.org/10.1590/1413-81232018231.05612015

6. Lemes EO, Fátima GG, Santos GCB, Costa JB. Análise do programa nacional de controle do tabagismo do ministério da saúde. Uniciências. 2017;21(2):86-92. doi: https://doi.org/10.17921/1415-5141.2017v21n2p86-92 7. Bazotti A, Finokiet M, Conti IL, França MTA, Waquil PD. Smoking and poverty in Brazil: an analysis of the profile of the smoking population based on the 200809 Brazilian government Family Budget Survey. Cienc Saúde Coletiva. 2016;21(1):45-52. doi: http://dx.doi. org/10.1590/1413-81232015211.16802014

8. Pérez-Romero S, Gascón-Cánovas JJ, SalmerónMartínez D, Parra-Hidalgo P, Monteagudo-Piqueras O. Características sociodemográficas y variabilidad geográfica relacionada con la satisfacción del paciente en Atención Primaria. Rev Calid Asist. 2016;31(5):3008. doi: https://doi.org/10.1016/j.cali.2016.01.004 9. Leal ACS, Silveira AC, Freire APCF, Santos APS, Ramos $D$, Ramos EMC, et al. Características sociodemográficas e padrão de consumo tabagístico de fumantes que procuram atendimento em programas de cessação. Mundo Saúde. (Impr.) 2017;41(2):163-9. doi: 10.15343/0104-7809.20174102163169

10. Ministério da Saúde. Instituto Nacional de Câncer José Alencar Gomes da Silva (BR). Secretaria-Executiva da Comissão Nacional para a Implementação da Convenção-Quadro Organização Mundial da Saúde para o Controle do Tabaco. Tabaco: uma ameaça ao desenvolvimento. Rio de Janeiro: Inca; 2017. [Acesso 12 maio 2019]. Disponível em: https:// 
www.inca.gov.br/publicacoes/notas-tecnicas/ tabaco-uma-ameaca-ao-desenvolvimento-0

11. Carmo HO, Santos GT. Atuação do enfermeiro frente as estratégias do programa nacional de controle ao tabagismo. Rev Rede Cuid Saúde. 2016;10(3):116. [Acesso 12 maio 2019]. Disponível em: http:// publicacoes.unigranrio.edu.br/index.php/rcs/article/ view/3053/2089

12. Silva TA, Ivo ML, Freitas SLF, Sales APA, Carvalho AMA. Smoking prevalence and of nicotine dependence's therapeutics: an integrative review. Rev Pesqui Cuid Fundam. 2016;8(4):4942-8. doi: http://dx.doi. org/10.9789/2175-5361.rpcfo.v8.3678

13. Rocha SAV, Hoepers ATC, Fröde TS, Steidle LJM, Pizzichini E, Pizzichini MMM. Prevalência de tabagismo e motivos para continuar a fumar: estudo de base populacional. J Bras Pneumol. 2019;45(4):e20170080. doi: https://doi.org/10.1590/1806-3713/e20170080

14. Bettio CJS, Bonilha LAS, Souza AS, Andrade SMO, Batiston AP. Emotional factors associated with smoking in individuals enrolled in a tobacco cessation program. Rev Bras Promoç Saúde. 2018;31(1):1-10. doi: http:// dx.doi.org/10.5020/18061230.2018.6536

15. Wittkowski L, Dias CRS. Avaliação dos resultados obtidos nos grupos de controle do tabagismo realizado numa unidade de saúde de Curitiba-PR. Rev Bras Med Fam Comum. 2017;12(39):1-11. doi: https://doi. org/10.5712/rbmfc12(39)1463

16. Müller EV, Silva MT, Grden CRB, Reche PM, Borges PKO, Sousa JAV. Factors associated with smoking among users of the family health strategy. Cogitare Enferm. 2017;(22)4:e50600. doi: http://dx.doi.org/10.5380/ ce.v22i4.50600

17. Silva LCC, Araújo AJ, Queiroz AMD, Sales MPU, Castellano MVC. Smoking control: challenges and achievements. J Bras Pneumol. 2016;42(4):290-8. doi: https://doi.org/10.1590/s1806-37562016000000145 18. Ministério da Saúde. Instituto Nacional de Câncer José Alencar Gomes da Silva (BR). Dados e números de prevalência do tabagismo [Internet]. 2019 [Acesso 24 ago 2019]. Disponível em: https://www.inca.gov.br/observatorioda-politica-nacional-de-controle-do-tabaco/ dados-e-numeros-prevalencia-tabagismo

19. Santos SR, Bitencourt ARC, Engel RH, Silva MHC, Stacciarini TSG, Cordeiro ALPC. Profile and factors associated with successful treatment of smokers in a specialized public service. Rev Enferm UERJ. 2018;26:e17381. doi: https://doi.org/10.12957/ reuerj.2018.17381

20. Pereira ES, Barreto GS. Fatores associados a cessação do tabagismo sem ajuda. REVA Acad Rev Cient Saúde. 2017;2(3):31-44. doi: 10.24118/ reva1806.9495.2.3.2017:347
21. Oliveira GMM, Mendes M, Dutra OP, Achutt A, Fernandes M, Azevedo V, et al. 2019: Recommendations for reducing tobacco consumption in Portuguesespeaking countries - positioning of the federation of Portuguese language cardiology societies. Arq Bras Cardiol. 2019;112(4):477-86. doi: https://doi. org/10.5935/abc.20190071

22. Santos MDV, Santos SV, Caccia-Bava MCGG. Prevalência de estratégias para cessação do uso do tabaco na atenção primária à saúde: uma revisão integrativa. Cienc Saúde Coletiva. 2019;24(2):563-72. doi: http:// dx.doi.org/10.1590/1413-81232018242.27712016

23. Paiva ARAB, Souza WA, Goyatá SLT, Junior LMS, Podestá MHMC, Ferreira EB. Grupo de apoio ao tabagista na estratégia de saúde da família: fatores de sucesso. Rev UninCor. 2017;15(2):436-48. doi: http://dx.doi. org/10.5892/ruvrd.v15i2.3249

24. Polonio IB, Oliveira M, Fernandes LMM. Tabagismo entre estudantes de medicina e enfermagem da universidade do Anhembi Morumbi: prevalência e avaliação da dependência nicotínica e escala de depressão e ansiedade. Arq Med Hosp Fac Ciênc Med Santa Casa São Paulo. [Internet]. 2017;62(1):12-7. [Acesso 17 maio 17 2019]. Disponível em: http:// arquivosmedicos.fcmsantacasasp.edu.br/index.php/ AMSCSP/article/view/86

25. França SAS, Neves ALF, Souza TAS, Martins NCN, Carneiro SR, Sarges ESNF, et al. Factors associated with smoking cessation. Rev Saúde Pública. 2015;49:10. doi: https://doi.org/10.1590/S0034-8910.2015049004946 26. Dornelles CF, Stumpf MAM, Nakamura MC, Kluthcovsky SC, Vernizi BT, Müller EV, el al. Estimativas de valores economizados com a cessação do tabagismo. RIES. 2018;7(2):100-9. doi: https://doi.org/10.33362/ ries.v7i2.1422

27. Devóglio LL, Corrente JE, Borgato MH, Godoy I. Smoking among female sex workers: prevalence and associated variables. J Bras Pneumol. 2017;43(1):6-13. doi: http://dx.doi.org/10.1590/ s1806-37562016000000162

28. Malta DC, Stopa SR, Santos MAS, Andrade SSCA, Oliveira TP, Cristo EB, et al. Evolution of tobacco use indicators according to telephone surveys, 2006-2014. Cad Saúde Pública. 2017;33(Suppl 3):e00134915. doi: https://doi.org/10.1590/0102-311×00134915.

29. Aguiar LM, Brasil LOF, Machado SHM, Monteiro DF, Santos GS, Brum CM, et al. Avaliação da dependência e tolerância à nicotina pelo teste de Fagerström em adultos tabagistas. Rev Conhecendo Online. 2016;3(1):30-43. [Acesso 17 maio 2019]. Disponível em: http://177.67.128.65/OJS/index.php/revista/ article/view/41 


\section{Contribuição dos Autores}

Concepção e planejamento do estudo: Lidia Dalgallo. Obtenção dos dados: Rafael da Rosa Maciel. Análise e interpretação dos dados: Rafael da Rosa Maciel, Lidia Dalgallo, Elaine Cristina Antunes Rinaldi. Análise estatística: Rafael da Rosa Maciel, Erildo Vicente Müller. Redação do manuscrito: Rafael da Rosa Maciel, Lidia Dalgallo, Elaine Cristina Antunes Rinaldi, Erildo Vicente Müller. Revisão crítica do manuscrito: Rafael da Rosa Maciel, Lidia Dalgallo, Elaine Cristina Antunes Rinaldi, Erildo Vicente Müller.

Todos os autores aprovaram a versão final do texto. Conflito de interesse: os autores declararam que não há conflito de interesse.

Esta licença permite que outros remixem, adaptem e criem a partir do seu trabalho para fins não comerciais, e embora os novos trabalhos tenham de lhe atribuir o devido crédito e não possam ser usados para fins comerciais, os usuários não têm de licenciar esses trabalhos derivados sob os mesmos termos. 